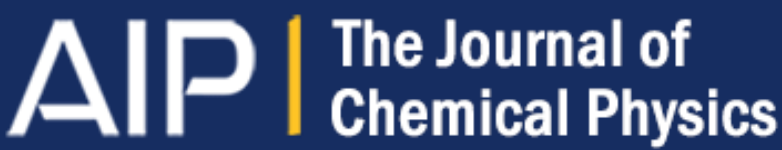

\section{A linked electron pair functional}

Peter J. Knowles and Bridgette Cooper

Citation: The Journal of Chemical Physics 133, 224106 (2010); doi: 10.1063/1.3507876

View online: http://dx.doi.org/10.1063/1.3507876

View Table of Contents: http://scitation.aip.org/content/aip/journal/jcp/133/22?ver=pdfcov

Published by the AIP Publishing

\section{AlP}




\title{
A linked electron pair functional
}

\author{
Peter J. Knowles ${ }^{\text {a) }}$ and Bridgette Cooper \\ School of Chemistry, Cardiff University, Main Building, Park Place, Cardiff CF10 3AT, United Kingdom
}

(Received 27 August 2010; accepted 11 October 2010; published online 14 December 2010)

\begin{abstract}
A modification of the variational configuration interaction functional in the first-order interacting space for molecular electronic structure is presented. The modified functional is a fully linked expression that by construction is extensive and invariant to transformations of the underlying orbital basis and is exact for an ensemble of separated two-electron subsystems. In addition, an approximation to variational coupled cluster is generated through truncation of the exponential cluster operator. When combined, these methods demonstrate accuracy that exceeds that of the standard coupledcluster method, in particular in situations where the reference Slater determinant is not a good approximation. ( ) 2010 American Institute of Physics. [doi:10.1063/1.3507876]
\end{abstract}

\section{INTRODUCTION}

The coupled-cluster (CC) method has in recent years emerged as the standard tool for computing molecular electronic structure. It has the advantage over variational configuration interaction (CI) that it is size extensive; although in the standard form where the time-independent Schrödinger equation is projected against a basis, its energy is not a variational upper bound to the exact energy. It has the advantage over many-body perturbation theory in that it is not troubled by considerations of convergence of the perturbation series, although it is often used in conjunction with perturbation theory for reasons of cost.

Despite the success and widespread adoption of CC, interest remains in alternative approaches. We have shown recently ${ }^{1}$ that in some strong correlation circumstances, such as the breaking of multiple bonds, coupled cluster with single and double excitations (CCSD) can fail, giving energies that are very much lower than full CI but variational optimization of the expectation value of the energy using the CCSD wavefunction can succeed. Although variational coupled-cluster theory is not a computationally feasible method, approximations to it may be useful.

Historically, simpler approaches were used before the emergence of coupled-cluster theory; these include linearized coupled-pair many-electron theory (LCPMET), ${ }^{2,3}$ the coupled-pair functional (CPF), ${ }^{4}$ and the coupled electron pair approximation (CEPA) ${ }^{5-7}$ More recent work $^{8}$ has resulted in more sophisticated, orbitally invariant approaches derived from the viewpoint of variational coupled-cluster theory. CC methodology for multideterminant reference functions is still an active research area, and in advance of a universally accepted and widely implemented multireference $\mathrm{CC}$, simple approximations are in widespread use. These include the average coupled-pair functional (ACPF), ${ }^{9}$ average quadratic coupled cluster (AQCC), ${ }^{10}$ quasidegenerate variational perturbation theory (QDVPT),${ }^{11}$ and the very simple Davidson correction. ${ }^{12}$ All these approaches can be viewed

\footnotetext{
a) Author to whom correspondence should be addressed. Electronic mail: KnowlesPJ@Cardiff.ac.uk.
}

as modifications of the standard variational method that attempt to eliminate unphysical unlinked cluster contributions that cause the CI energy to scale incorrectly with the number of electrons.

A quite different approach, but resulting in similar formulations, is taken by Kollmar ${ }^{13}$ and Mazziotti. ${ }^{14-16}$ Here, the emphasis is on constructing a simple energy functional that by construction satisfies, or nearly satisfies, the known subset of conditions for $N$-representability of the two-electron reduced density matrix. The most recent of these functionals ${ }^{15}$ produces results that are extremely impressive; the method has less complexity than CCSD but accuracy that rivals CC including triple excitations (CCSDT).

This paper presents a new family of functionals constructed from the wavefunction perspective but showing similar accuracy. They have the additional advantage of being strict tensor quantities, which are therefore invariant to transformations of the underlying orbital space, eliminating the necessity to rely on a particular canonicalization choice of those orbitals to define the ansatz and the potential breakdown of extensivity if the prescribed orbitals are not spatially local.

\section{METHOD}

The standard configuration interaction energy expression can be written in intermediate normalization as

$$
\begin{aligned}
E & =\frac{\left\langle 0\left|\left(1+\hat{C}^{\dagger}\right) \hat{H}(1+\hat{C})\right| 0\right\rangle}{\left\langle 0\left|\left(1+\hat{C}^{\dagger}\right)(1+\hat{C})\right| 0\right\rangle} \\
& =\frac{\langle 0|\hat{H}| 0\rangle+2\langle 0|\hat{H} \hat{C}| 0\rangle+\left\langle 0\left|\hat{C}^{\dagger} \hat{H} \hat{C}\right| 0\right\rangle}{1+\left\langle 0\left|\hat{C}^{\dagger} \hat{C}\right| 0\right\rangle},
\end{aligned}
$$

where $|0\rangle$ is a suitable reference wavefunction (assumed here to be a single Slater determinant built from real orbitals $\left.\left\{\psi_{i}, i=1,2, \ldots N\right\}\right)$ and $\hat{C}$ is an operator that generates single and double orbital excitations into virtual orbitals denoted $\left\{\psi_{a}\right\}$,

$$
\hat{C}=\hat{C}_{1}+\hat{C}_{2}=C_{a}^{i} a^{\dagger} i+\frac{1}{4} C_{a b}^{i j} a^{\dagger} b^{\dagger} j i,
$$

assuming the usual Einstein summation convention. The restriction to single and double excitations arises from 
perturbational considerations; the first-order perturbed wavefunction for a two-body hamiltonian lies within the space spanned by $\left(\hat{C}_{1}+\hat{C}_{2}\right)|0\rangle$.

Equation (1) is an upper bound to the exact energy and can thus be used in a variational calculation, but unfortunately the energy expression is unphysical, leading to an energy that does not scale linearly with the number of electrons and, in particular, not satisfying the requirement that a calculation on an ensemble of separated subsystems yields the sum of the subsystem energies. This lack of extensivity renders the performance of this single- and double-excitation CI (CISD) approach very poor except for a small number of electrons.

An extensive, or approximately extensive, functional can be obtained by avoiding division by the complete denominator, which grows with system size. Each term in the numerator can be scaled with just those parts of the denominator that are local, according to some scheme (for example, CPF (Ref. 4) and Kollmar's functional ${ }^{13}$ ). The CEPA approach achieves a similar effect by eliminating nonlocal unlinked cluster contributions from the working equations. ${ }^{6}$ In the ACPF method, the functional is modified by simply dividing $\left\langle 0\left|\hat{C}^{\dagger} \hat{C}\right| 0\right\rangle$ by the number of electron pairs to render the denominator approximately intensive. All these approaches produce results that usually approximate CCSD quite well but (apart from $\mathrm{ACPF}$ ) require a particular choice of molecular orbitals in order to uniquely define the partitioning of unlinked cluster contributions. It would be much better to have a formalism that was a fully linked tensor expression, invariant to such transformations.

Such a functional may be achieved as follows. Ignoring in the first instance single orbital excitations, the following identities set the scene for a pairwise partitioning of the doubleexcitation configuration interaction (CID) wavefunction:

$$
\begin{aligned}
& \frac{1}{2} i i^{\dagger} \hat{C}_{2}|0\rangle=\hat{C}_{2}|0\rangle, \\
& \frac{1}{2} i j j^{\dagger} i^{\dagger} \hat{C}_{2}|0\rangle=\hat{C}_{2}|0\rangle, \\
& \text { i.e., }\left(\frac{1}{2}(1-\lambda) i i^{\dagger}+\frac{\lambda}{2} i j j^{\dagger} i^{\dagger}\right) \hat{C}_{2}|0\rangle=\hat{C}_{2}|0\rangle,
\end{aligned}
$$

where $\lambda$ can take any value. The identities can be justified by realizing that $\hat{C}_{2}|0\rangle$ is a linear combination of determinants that have two Hartree-Fock occupied orbitals vacant, and each of these determinants will be found and converted to a determinant with all HF orbitals occupied by exactly one of the unique double creators $j^{\dagger} i^{\dagger}, i>j$; the double annihilator $i j$ then restores the original $N$-electron doubly excited determinant.

In terms of the one- and two-hole reduced density matrices, ${ }^{17}$

$$
\begin{aligned}
& \eta_{k l}^{i j}=\left\langle 0\left|\hat{C}^{\dagger} k l j^{\dagger} i^{\dagger} \hat{C}\right| 0\right\rangle=\frac{1}{2} C_{a b}^{i j} C_{a b}^{k l}, \\
& \eta_{k}^{i}=\left\langle 0\left|\hat{C}^{\dagger} k i^{\dagger} \hat{C}\right| 0\right\rangle=\frac{1}{2} C_{a b}^{i j} C_{a b}^{k j} \equiv \eta_{k j}^{i j},
\end{aligned}
$$

we analogously define

$$
\Delta_{k l}^{i j}=\lambda \eta_{k l}^{i j}+\frac{1}{2}(1-\lambda)\left(\delta_{k}^{i} \eta_{l}^{j}+\delta_{l}^{j} \eta_{k}^{i}-\delta_{l}^{i} \eta_{k}^{j}-\delta_{k}^{j} \eta_{l}^{i}\right) .
$$

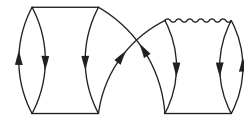

A

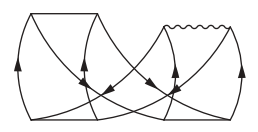

$\mathrm{C}$

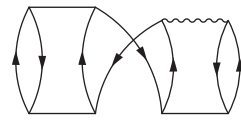

B

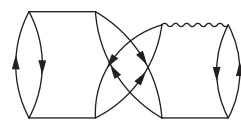

$\mathrm{D}$
FIG. 1. Diagrams contributing to $\left\langle 0\left|\hat{T}_{2}^{\dagger} \hat{H} \hat{T}_{2}^{2}\right| 0\right\rangle$.

In the case of $\lambda=1$, the normalization integral forming the CI denominator is then

$$
1+\left\langle 0\left|\hat{C}^{\dagger} \hat{C}\right| 0\right\rangle=1+\frac{1}{2} \Delta_{i j}^{i j}=1+\operatorname{tr}(\boldsymbol{\Delta}),
$$

where $\boldsymbol{\Delta}$ is a two-index matrix with rows and columns labeled by the composite indices $\{i j, i=2, \ldots, N, j=1, \ldots$, $i-1\}$. We also define $U_{k l}^{i j}=\delta_{k l}^{i j}+\Delta_{k l}^{i j}$, and with this definition, matrix powers appear as

$$
\left(\boldsymbol{U}^{2}\right)_{k l}^{i j}=\frac{1}{2} U_{m n}^{i j} U_{k l}^{m n}
$$

Note that because the $\eta$ matrices are positive definite, so are $\Delta$ and $\boldsymbol{U}$, and therefore matrix powers are real and single-valued for real as well as integer powers. We then introduce partial local normalization into the configuration mixing coefficients in the numerator through transformations of the form

$$
{ }_{q} C_{a b}^{i j}=\frac{1}{2}\left(\boldsymbol{U}^{-q / 2}\right)_{m n}^{i j} C_{a b}^{m n} \equiv\left(\boldsymbol{U}^{-q / 2} \boldsymbol{C}\right)_{a b}^{i j} .
$$

The idea is that this introduces division by those parts of the denominator in the CI quotient that are local to a particular part of the numerator in the sense that the contribution from $\boldsymbol{C}^{k l}$ in the denominator to terms involving $\boldsymbol{C}^{i j}$ in the numerator is governed by the size of $\Delta_{i j, k l}$. In the extreme case of separated subsystems with localized orbitals, the relevant elements of $\boldsymbol{U}^{-q / 2}$ will be zero, allowing proper additive separation of energies.

The proposed linked pair energy functional (LPF), assuming real orbitals, is then

$$
E=E_{0}+2\left\langle 0\left|{ }_{2} \hat{C}^{\dagger} \hat{H}\right| 0\right\rangle+\left\langle 0\left|{ }_{1} \hat{C}^{\dagger}\left(\hat{H}-E_{0}\right){ }_{1} \hat{C}\right| 0\right\rangle,
$$

where $E_{0}=\langle 0|\hat{H}| 0\rangle$. This functional has the following features.

1. It is exactly equivalent to CID in the case of two electrons, since in that case $\boldsymbol{U}$ is one-dimensional and equal to the norm of the wavefunction, $1+\left\langle\hat{C}^{\dagger} \hat{C}\right\rangle$.

2. For more electrons, the effect of the matrix inverse is to introduce division by those parts of the denominator that are coupled to the correlation of a given pair of reference-state orbitals rather than by the complete denominator.

3. The expression can be written in terms of linked diagrams and is therefore rigorously extensive. 
TABLE I. Potential energy function for hydrogen fluoride. The 6-31G** (Refs. 22 and 23) orbital basis set is used, and excitations from the spin-restricted Hartree-Fock $1 \sigma$ orbital excluded. Results at different bond lengths $R$ are compared with full configuration interaction (FCI) energies given in Hartree units $\left(E_{h}\right)$.

\begin{tabular}{|c|c|c|c|c|c|c|c|c|c|}
\hline \multirow[b]{2}{*}{$R(\AA)$} & Energy $\left(E_{h}\right)$ & \multicolumn{8}{|c|}{ Error $\left(\mathrm{m} E_{h}\right)$} \\
\hline & FCI & $\mathrm{HF}$ & CCSD & CCSDT & CEPA(1) & LPF & QVCCD & $\mathrm{LPF}+\mathrm{QVCCD}$ & VCCD \\
\hline 0.9 & -100.2011 & 189.4 & 2.5 & 0.3 & 2.9 & 5.0 & 2.2 & 2.2 & 2.2 \\
\hline 1.4 & -100.1073 & 216.7 & 4.7 & 0.6 & 3.8 & 7.6 & 4.3 & 4.0 & 4.1 \\
\hline 1.8 & -100.0389 & 251.0 & 9.0 & 1.0 & 4.5 & 10.5 & $\mathrm{a}$ & 7.5 & 7.7 \\
\hline 2.2 & -100.0095 & 296.1 & 14.8 & 1.2 & 5.8 & 13.3 & & 12.5 & 12.6 \\
\hline 2.6 & -100.0005 & 339.2 & 19.0 & 1.1 & 7.2 & 14.9 & & 16.8 & 16.4 \\
\hline 2.8 & -99.9990 & 357.2 & 20.3 & 1.0 & 7.8 & 15.5 & & 18.4 & 17.6 \\
\hline $\mathrm{F}, \mathrm{H}$ atoms & -99.9973 & 137.3 & 1.4 & 0.2 & 1.2 & $1.2^{\mathrm{b}}$ & & & \\
\hline$R_{e}(\AA)$ & 0.9215 & -0.0210 & -0.0011 & -0.0003 & -0.0007 & -0.0018 & -0.0008 & -0.0008 & -0.0008 \\
\hline$\omega_{e}\left(\mathrm{~cm}^{-1}\right)$ & 4171.0 & +322.0 & +21.5 & -4.9 & +13.6 & +32.2 & +19.2 & +17.3 & +18.7 \\
\hline
\end{tabular}

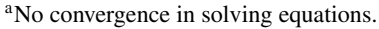

${ }^{\mathrm{b}}$ For technical reasons, spin-unrestricted Hartree-Fock, rather than Brueckner CCD, orbitals were used.

4. The energy is a scalar that is invariant to rotations in the underlying spaces $\left\{\psi_{i}\right\}$ and $\left\{\psi_{a}\right\}$.

5. The energy is not an upper bound to the exact groundstate Schrödinger eigenvalue, but variational minimization of the functional gives a theory that satisfies the symmetric generalized Hellmann-Feynman theorem. ${ }^{18}$

The functional has a formal relationship to coupledcluster theory, through consideration of the variational coupled cluster (VCC) ansatz,

$$
\begin{aligned}
E_{\mathrm{VCC}} & =\frac{\left\langle 0\left|\exp \left(\hat{T}^{\dagger}\right) \hat{H} \exp (\hat{T})\right| 0\right\rangle}{\left\langle 0\left|\exp \left(\hat{T}^{\dagger}\right) \exp (\hat{T})\right| 0\right\rangle} \\
& =\left\langle 0\left|\exp \left(\hat{T}^{\dagger}\right) \hat{H} \exp (\hat{T})\right| 0\right\rangle_{\text {linked }}
\end{aligned}
$$

The expansion of $E_{\mathrm{VCC}}$ as a series of linked diagrams is nonterminating, but for coupled cluster with only double excitation. (CCD operators, i.e. $\hat{T}=\hat{T}_{2}$ ), a subset of these diagrams

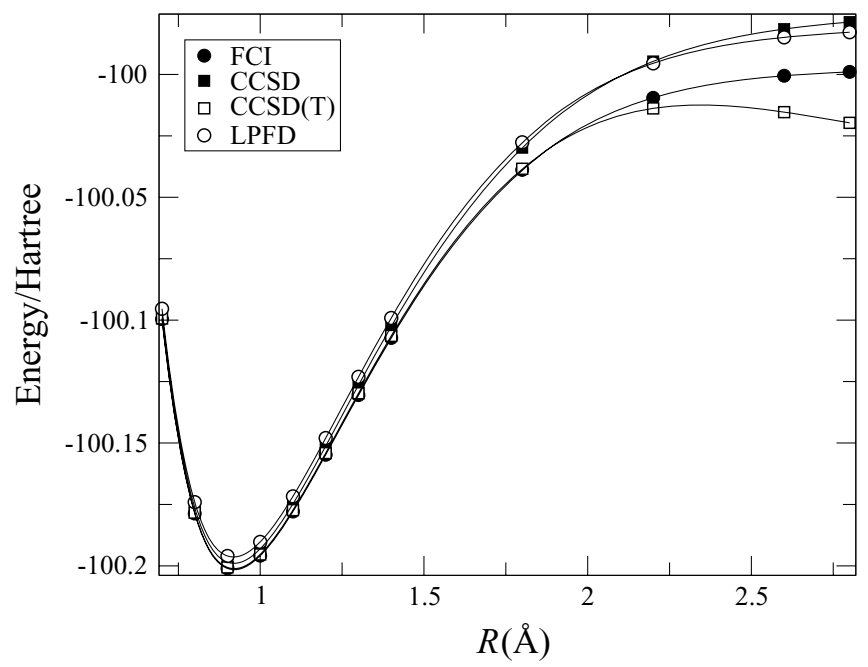

FIG. 2. Potential energy curve of HF with $6-31 \mathrm{G}^{* *}$ basis set. is identical to the series expansion of Eq. (13), provided that $\lambda=-1$. This leads to the conclusion that this value of $\lambda$ is the only one that should be used, and we adopt it henceforth. Furthermore, for two electrons, the two series are identical, since both are exact. This is illustrated for the $\mathcal{O}\left(\hat{T}^{3}\right)$ terms occurring in VCCD in Fig. 1. For two electrons or any number of isolated two-electron subsystems, but not otherwise, diagrams $\mathrm{A}$ and $\mathrm{D}$ (Fig. 1) cancel each other. The remaining diagrams, B and C (Fig. 1), are equal to the third-order part of the LPF [Eq. (13)]. These correspondences occur at all powers of $\hat{T}$. Thus the LPF can be viewed as an approximation to VCCD in which all diagrams that contribute in the case of two electrons are included to infinite order but the remainder are omitted.

The computational implementation of the variational method applied to the LPF defined by Eq. (14) follows by straightforward differentiation of the functional with respect to the coefficients $C_{a b}^{i j}$ to form a residual vector that is used in a standard preconditioned relaxation scheme with the direct inversion in the iterative subspace (DIIS) accelerator. ${ }^{19}$ This implicates the construction of $\boldsymbol{U}^{-1 / 2}, \boldsymbol{U}^{-1}, \boldsymbol{U}^{-3 / 2}$, and $\boldsymbol{U}^{-2}$ together with a number of subsequent transformations of the Eq. (12). In our present implementation, the matrix powers are evaluated by first diagonalizing $\boldsymbol{U}$. Thus relative to the parent CID method, there are additional $O\left(N^{6}\right)$ linear algebra and

TABLE II. Potential energy curves for abstracting a hydrogen from $\mathrm{CH}_{4}$ with $6-31 \mathrm{G}^{*}$ basis set, with the remaining bond lengths fixed at $1.86 \AA$. FCI values given in Hartrees and other methods as energy errors in milliHartrees in comparison to the $\mathrm{FCI}$ values.

\begin{tabular}{lccrrrrr}
\hline \hline$R$ & FCI & RHF & CCSD & CCSD(T) & BCCD & LPF & CEPA(1) \\
\hline 1.1 & -40.3562 & 161.2 & 3.3 & 0.4 & 3.5 & 4.5 & 2.6 \\
1.6 & -40.2891 & 173.2 & 4.7 & 0.6 & 5.2 & 6.2 & 3.3 \\
2.2 & -40.2146 & 206.0 & 9.1 & 0.4 & 9.8 & 10.0 & 4.5 \\
2.8 & -40.1869 & 254.6 & 14.7 & -4.2 & 14.9 & 13.8 & 5.2 \\
3.2 & -40.1817 & 286.0 & 17.1 & -9.9 & 16.9 & 15.1 & 5.3 \\
3.4 & -40.1806 & 299.8 & 17.8 & -12.7 & 17.5 & 15.5 & 5.2 \\
\hline \hline
\end{tabular}




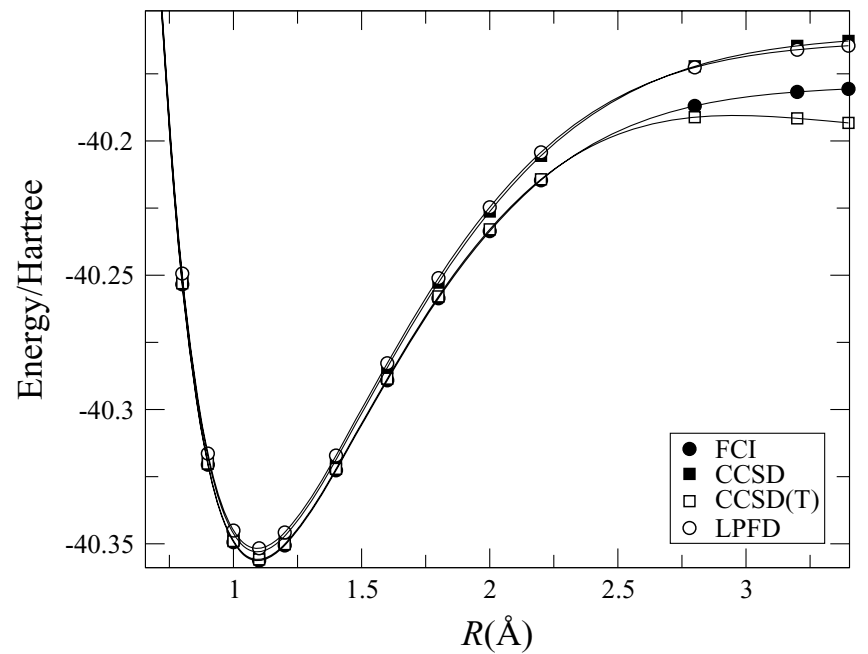

FIG. 3. Potential energy for hydrogen abstraction from $\mathrm{CH}_{4}$ with $6-31 \mathrm{G}^{*}$ basis set.

$O\left(N^{4} m^{2}\right)$ matrix multiplication operations ( $N$ electrons, $m$ orbital basis functions). The method is less expensive and less complicated than CCD, where there are additional $O\left(N^{3} \mathrm{~m}^{3}\right)$ terms arising from $\left(\hat{H} \hat{T}^{2}\right)_{c}|0\rangle{ }^{20}$

An alternative approach to approximating VCC is to truncate at some finite power of $\hat{T}$. The expectation value CC (XCC) ansatz ${ }^{21}$ takes all terms in the linked expansion of Eq. (14) up to a given power; this method is known to be slowly convergent. An alternative is to replace $\exp (\hat{T})$ by $1+\hat{T}$ in the linked expansion; this is the LCPMET/CEPA- 0 ansatz. We here consider an extension of this approach that we term quadratic variational coupled cluster (QVCC), with

$$
E_{\mathrm{QVCC}}=\left\langle 0\left|\left(1+\hat{T}^{\dagger}+\frac{1}{2}\left(\hat{T}^{\dagger}\right)^{2}\right) \hat{H}\left(1+\hat{T}+\frac{1}{2} \hat{T}^{2}\right)\right| 0\right\rangle_{\text {linked }} .
$$

In this work, we restrict attention to the double-excitation case, $\hat{T}=\hat{T}_{2}$ (VCCD and QVCCD), but in principle one should include single and higher excitation operators too, although this would be extremely complicated and expensive at the level of triple excitations. Like LCPMET and XCC, this functional is not expected to be suitable for accurate work except when $\hat{T}$ is small, since it is not exact even for two electrons. However, it can be combined with the LPF to construct an energy functional which is the sum of LPF and QVCCD but omitting duplication of any common diagrams. This re- stores the possibility of use in the strong correlation regime. The additional terms in QVCCD are not all easy to compute, with some of them showing a cost scaling that is sixth order in the number of virtual orbitals, but it may form a starting point for approximations similar in spirit to the LPF.

One may attempt to compare QVCC with XCC, VCC, and full $\mathrm{CI}$ through a perturbational analysis that assumes a Fock zero-order hamiltonian $\hat{H}^{(0)}$. QVCCD first differs from VCCD at sixth order through the omission of $\langle 0|\left(\hat{T}_{2}^{\dagger}\right)^{2}$ $\left(\hat{H}-\hat{H}^{(0)}\right) \hat{T}_{2}^{3}|0\rangle_{\text {linked }}$ and $\left\langle 0\left|\left(\hat{T}_{2}^{\dagger}\right)^{3} \hat{H}^{(0)} \hat{T}_{2}^{3}\right| 0\right\rangle_{\text {linked. }}$ QVCC with single and double excitations (QVCCSD) would, like all double-excitation theories, deviate from full $\mathrm{CI}$ at fourth order because of the omission of terms involving triple excitations, e.g., $\left\langle 0\left|\hat{T}_{3}^{\dagger} \hat{H}^{(0)} \hat{T}_{3}\right| 0\right\rangle_{\text {linked. }}$. However it agrees with single- and double-excitation VCC (VCCSD) to fifth order, since the lowest-order contribution arising from the omitted $\hat{T}^{3}$ would not involve the second-order single excitation operator.

In this initial exploration of the functionals, Brueckner orbitals obtained from a preceding Brueckner coupled cluster with only double excitations (BCCD) (Ref. 20) calculation have been used and the (small) effects of single excitations ignored. Future work will explore other approaches to single excitations and also the use of multideterminant reference functions.

\section{EXAMPLES}

Table I and Fig. 2 illustrate the performance of the functional on the potential energy curve of the HF molecule. Close to the equilibrium bond length, the error in the energy is a few milliHartrees, comparable with that of CCSD. At longer bond lengths, however, the LPF approximation appears to degrade somewhat less than standard single-reference CCSD. This behavior is similar to that observed with both CEPAlike approximations and with functionals derived from $\mathrm{N}$ representability considerations. The $+\mathrm{QVCCD}$ correction reduces the absolute error near equilibrium, but not at longer bond lengths.

The potential energy curves obtained when a single hydrogen atom is abstracted from $\mathrm{CH}_{4}$ are shown in Fig. 3 and Table II. Calculations were performed using the $6-31 \mathrm{G}^{*}$ basis set. These results show the same trends as for HF in that the errors at the equilibrium bond length are just a few milliHartrees and are comparable to CCSD. At longer bond lengths the LPF errors have increased to $15 \mathrm{mHartree}$; however, it does perform slightly better than CCSD.

TABLE III. Equilibrium energies, bond lengths, and harmonic vibrational wavenumbers are presented for $\mathrm{HF}, \mathrm{F}_{2}$, and $\mathrm{CO}$ in cc-pVQZ basis set.

\begin{tabular}{|c|c|c|c|c|c|c|c|c|c|}
\hline \multirow[b]{2}{*}{ Method } & \multicolumn{3}{|c|}{$\mathrm{HF}$} & \multicolumn{3}{|c|}{$\mathrm{F}_{2}$} & \multicolumn{3}{|c|}{$\mathrm{CO}$} \\
\hline & Energy $\left(E_{h}\right)$ & $R_{e}(\AA)$ & $\omega_{e}\left(\mathrm{~cm}^{-1}\right)$ & Energy $\left(E_{h}\right)$ & $R_{e}(\AA)$ & $\omega_{e}\left(\mathrm{~cm}^{-1}\right)$ & Energy $\left(E_{h}\right)$ & $R_{e}(\AA)$ & $\omega_{e}\left(\mathrm{~cm}^{-1}\right)$ \\
\hline CISD & -100.3554 & 0.9105 & 4259 & -199.2986 & 1.3660 & 1116 & -113.1447 & 1.1182 & 2288 \\
\hline CCSD & -100.3654 & 0.9137 & 4205 & -199.3383 & 1.3906 & 1016 & -113.1694 & 1.1243 & 2234 \\
\hline BCCD & -100.3650 & 0.9134 & 4211 & -199.3373 & 1.3892 & 1021 & -113.1682 & 1.1231 & 2249 \\
\hline K & -100.3657 & 0.9140 & 4195 & -199.3417 & 1.3984 & 974 & -113.1725 & 1.1256 & 2217 \\
\hline M & -100.3691 & 0.9153 & 4174 & -199.3520 & 1.4082 & 934 & -113.1807 & 1.1288 & 2183 \\
\hline LPF & -100.3625 & 0.9126 & 4223 & -199.3349 & 1.3941 & 989 & -113.1652 & 1.1219 & 2262 \\
\hline CEPA(1) & -100.3660 & 0.9142 & 4190 & -199.3449 & 1.4066 & 941 & -113.1740 & 1.1268 & 2202 \\
\hline
\end{tabular}




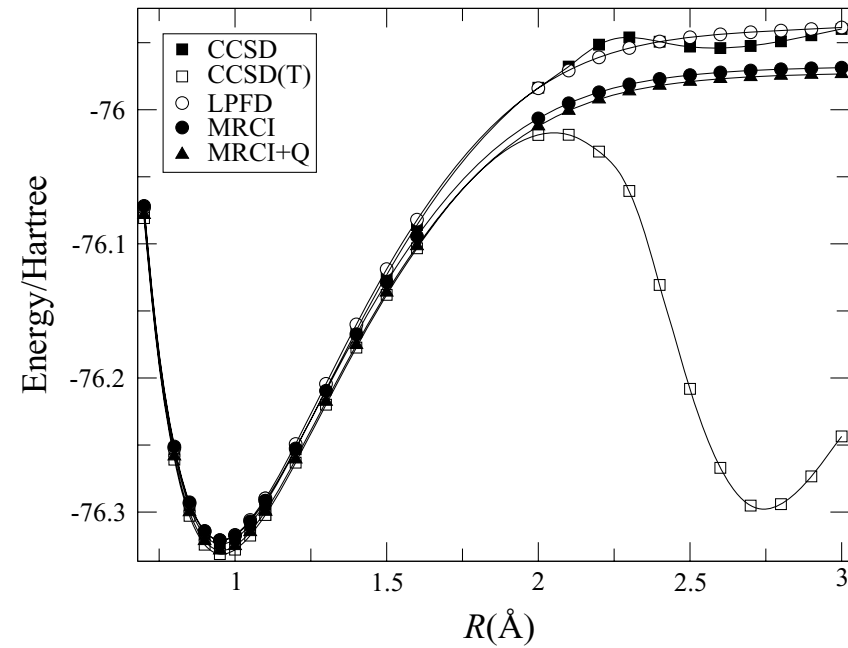

FIG. 4. Potential energy for symmetric stretching of $\mathrm{H}_{2} \mathrm{O}$ with cc-pVTZ basis set.

Figure 4 illustrates the potential energy curve along the symmetric stretching coordinate of $\mathrm{H}_{2} \mathrm{O}$ in the cc-pVTZ orbital basis set. ${ }^{24}$ The angle between the $\mathrm{O}-\mathrm{H}$ bonds was fixed at $107.6^{\circ}$. This is a more demanding case in comparison to the single bond breaks in $\mathrm{HF}$ and $\mathrm{CH}_{4}$, as it involves the simultaneous breaking of both the $\mathrm{O}-\mathrm{H}$ bonds. The LPF again at equilibrium bond length performs comparably with CCSD. At long bond lengths LPF performs somewhat better than CCSD, in particular not showing the same unphysical maximum potential energy as CCSD at around $2.3 \AA$.

Equilibrium energies, bond lengths, and harmonic frequencies for $\mathrm{HF}, \mathrm{F}_{2}$, and $\mathrm{CO}$ calculated with the cc-pVQZ basis set ${ }^{24}$ are presented in Table III. For each of the diatomic molecules, energies were calculated at five points around the equilibrium bond length, and the spectroscopic quantities were obtained by interpolating a fourth-order polynomial. The results show that the LPF overbinds in comparison to CCSD for $\mathrm{HF}$ and $\mathrm{F}_{2}$, as is indicated by a shorter equilibrium

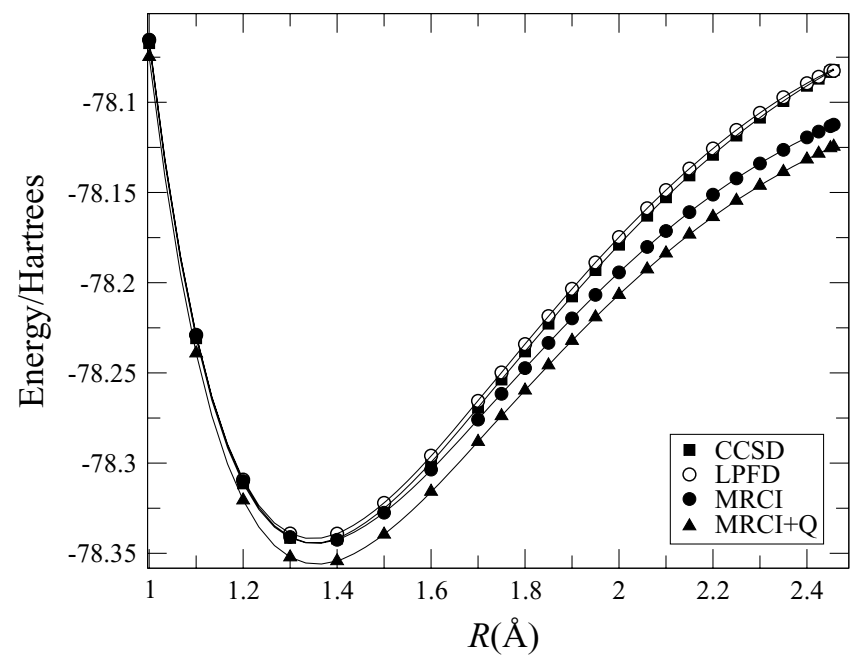

FIG. 5. Potential energy for ethene, cc-pVDZ basis, for stretching of carboncarbon bond. bond length, $R_{e}$, and higher harmonic wavenumber, $\omega_{e}$. However, for CO the LPF underbinds in comparison to CCSD.

Figure 5 shows the potential energy for the ethene molecule as a function of the carbon-carbon bond length, with the $\mathrm{C}-\mathrm{H}$ bond lengths and angles kept fixed and in the ccpVDZ basis. In this case, the LPF method closely mimics the performance of CCSD as the double bond is broken, with the results, as expected, being somewhat worse than those from internally contracted multireference configuration interaction (MRCI) (Ref. 25) and Davidson-corrected ${ }^{12}$ (MRCI+Q) calculations.

\section{CONCLUSIONS}

We have shown that it is possible to construct an energy functional in the spirit of CEPA and related methods-a simple modification of the variational configuration interaction ansatz-that simultaneously is extensive, is invariant to orbital rotations, is exact for two-electron subsystems, and exhibits an accuracy that is comparable to that of VCCSD. The structure of the approximation gives insight into the nature of the electron correlation effect in molecules: the energy contributions in full coupled cluster that cancel in the case of two electrons appear also to be relatively unimportant in manyelectron molecules.

\section{ACKNOWLEDGMENTS}

This work was performed using the computational facilities of the Advanced Research Computing @ Cardiff (ARCCA) Division, Cardiff University, and was supported in part by EPSRC.

${ }^{1}$ B. Cooper and P. J. Knowles, "Benchmark studies of variational, unitary, and extended coupled cluster methods," J. Chem. Phys. (in press).

${ }^{2}$ R. Ahlrichs, Comput. Phys. Commun. 17, 31 (1979).

${ }^{3}$ S. Koch and W. Kutzelnigg, Theor. Chim. Acta 59, 387 (1981).

${ }^{4}$ R. Ahlrichs, P. Scharf, and C. Ehrhardt, J. Chem. Phys. 82, 890 (1985).

${ }^{5}$ H. P. Kelly, Phys. Rev. 134, A1450 (1964).

${ }^{6}$ W. Meyer, J. Chem. Phys. 58, 1017 (1973).

${ }^{7}$ P. Pulay and S. Sæb $\varnothing$, Chem. Phys. Lett. 117, 37 (1985).

${ }^{8}$ M. Nooijen and R. J. LeRoy, J. Mol. Struct.: THEOCHEM, 768, 25 (2006).

${ }^{9}$ R. J. Gdanitz and R. Ahlrichs, Chem. Phys. Lett. 143, 413 (1988).

${ }^{10}$ P. G. Szalay and R. J. Bartlett, Chem. Phys. Lett. 214, 481 (1993)

${ }^{11}$ R. J. Cave and E. R. Davidson, J. Chem. Phys. 89, 6798 (1988).

${ }^{12}$ S. R. Langhoff and E. R. Davidson, Int. J. Quantum. Chem. 8, 61 (1974).

${ }^{13}$ C. Kollmar, J. Chem. Phys. 125, 084108 (2006).

${ }^{14}$ D. A. Mazziotti, Acc. Chem. Res. 39, 207 (2006).

${ }^{15}$ D. A. Mazziotti, Phys. Rev. Lett. 101, 253002 (2008).

${ }^{16}$ D. A. Mazziotti, Phys. Rev. A 81, 062515 (2010).

${ }^{17}$ W. Kutzelnigg and D. Mukherjee, J. Chem. Phys. 110, 2800 (1999).

${ }^{18}$ P. G. Szalay, M. Nooijen, and R. J. Bartlett, J. Chem. Phys. 103, 281 (1995).

${ }^{19}$ P. Pulay, Chem. Phys. Lett. 73, 393 (1980) .

${ }^{20}$ C. Hampel, K. A. Peterson, and H.-J. Werner, Chem. Phys. Lett. 190, 1 (1992).

${ }^{21}$ R. J. Bartlett and J. Noga, Chem. Phys. Lett. 150, 29 (1988).

${ }^{22}$ W. J. Hehre, R. Ditchfield, and J. A. Pople, J. Chem. Phys. 56, 2257 (1972).

${ }^{23}$ P. C. Hariharan and J. A. Pople, Theor. Chim. Acta 28, 213 (1973).

${ }^{24}$ T. H. Dunning, Jr., J. Chem. Phys. 90, 1007 (1989).

${ }^{25}$ H.-J. Werner and P. J. Knowles, J. Chem. Phys. 89, 5803 (1988). 